\title{
Evaluating Mobility Support in ZigBee Networks ${ }^{\star}$
}

\author{
Tony Sun ${ }^{1}$, Nia-Chiang Liang ${ }^{1}$, Ling-Jyh Chen ${ }^{2}$, Ping-Chieh Chen ${ }^{1}$, \\ and Mario Gerla ${ }^{1}$ \\ ${ }^{1}$ Department of Computer Science, University of California at Los Angeles \\ ${ }^{2}$ Institute of Information Science, Academia Sinica
}

\begin{abstract}
The deployment of ZigBee networks is expected to facilitate numerous applications, such as home healthcare, medical monitoring, consumer electronics, and environmental sensors. For many envisioned applications, device mobility is unavoidable and must be accommodated. Therefore, providing ubiquitous connection to/from a mobile ZigBee device is crucial for future ZigBee applications. In particular, knowledge of how nodal mobility affects ZigBee routing protocol is of significance. In this paper, our contributions are twofold. First, we dissect ZigBee routing and its support for device mobility, and we analyzed the current provisions in dealing with different mobility cases. Second, we performed a rich set of preliminary tests, illustrating the inefficacy of current standard. Our results indicate that ZigBee device type plays a significant role in determining the routing performance in most mobile scenarios.
\end{abstract}

Keywords: Mobility, Routing, ZigBee, IEEE 802.15.4, Simulation.

\section{Introduction}

With wireless networking technologies permeating into the very fabrics of our working and living environment, simple appliances and numerous traditional wired services can now be efficiently connected wirelessly. This provides simple yet effective control/monitoring conveniences, while allowing very interesting applications to be developed on top of these wireless network enabled gadgets. The ZigBee standard [2], designed to interconnect simple devices, is the latest attempt to realize this wireless network vision. In the context of a business environment, this wireless technology can facilitate better automated control/management of facilities and assets. Additionally, there are also many ZigBee applications for home-appliance networks, home healthcare, medical monitoring, consumer electronics, and environmental sensors.

For an environment richly connected with ZigBee devices, drastic topological changes can occur due to device failures, mobility, and other factors. For certain applications, device mobility is unavoidable. For example, a health monitoring application for the elderly described in [4] [3], where a ZigBee enabled health monitoring sensor alerts the hospital, through an adjacent network, when a health-related emergency has occurred. The consequence is disastrous if the message was not delivered as intended. Therefore, understanding the performance of ZigBee networks becomes

* This work was co-sponsored by the National Science Council and the National Science Foundation under grant numbers NSC 95-2218-E-002-072 and ANI-0335302.

T.-W. Kuo et al. (Eds.): EUC 2007, LNCS 4808, pp. 87-100, 2007.

(c) IFIP International Federation for Information Processing 2007 
important in determining the applicability of many applications. In particular, knowledge of how nodal mobility affects the workings of the ZigBee routing protocols is of significance.

Without a doubt, mobility support is important to the proper functioning of many envisioned ZigBee applications. Since mobility is anticipated and unavoidable, adequate mobility support is important in ensuring ubiquitous connection to/from the mobile devices. In this study, our contribution is twofold. First, we dissected ZigBee routing and its current support for device mobility. It is the goal of this study to identify the existing provisions in accommodating ZigBee device mobility, and to analyze the adequacy of these provisions in dealing with different mobility cases. Secondly, we ran a rich set of preliminary simulations, illustrating the inefficacy of current standard in handling mobility. Our results reveal that existing ZigBee provisions for mobility is inadequate, and mobility problem was not thoroughly considered by the standard. Moreover, we found that the current recovery mechanisms are not reliable, or responsive enough in all mobility cases. Finally, we found that the situation worsens when there are multiple instances of mobility in the ZigBee network, yet routing performance in ZigBee network is closely tied to the ZigBee node types used.

The rest of this paper is organized as follows. In section 2, we summarize the IEEE 802.15.4 and the ZigBee specifications. Section 3 discusses the routing and address allocation mechanism deployed in ZigBee mesh routing, and analyzes the response of the routing protocol in basic mobility cases. Section 4 is an equitable treatment to the ZigBee tree routing mechanism, where the behavior of tree routing is explained and analyzed for basic mobility scenarios. Section 5 presents a rich set of preliminary simulation results. Finally, in section 6 , we discuss the tradeoff between the two routing mechanism in dealing with mobility and conclude the paper.

\section{Overview}

\subsection{IEEE 802.15.4}

Based on the PHY and MAC layers specified by IEEE 802.15.4 WPAN standard [1], the ZigBee specification establishes the framework for the Network and Application layers. Specifically,at the MAC layer, IEEE 802.15.4 controls access to the radio channel using the Carrier Sense Multiple Access with Collision Avoidance (CSMA/CA) or the optional slotted CSMA/CA mechanism, as respectively utilized by the beaconless and beaconed modes.

Two device types are specified within the IEEE 802.15.4 framework: full function device (FFD) and reduced function device (RFD). An FFD generally have more responsibility in that they must maintain routing tables, participate in route discovery and repair, maintain beaconing framework, and handle node joins. Moreover, a FFD have the capability of communicating with any other devices within its transmission range. On the other hand, a RFD simply maintains the minimum amount of knowledge to stay on the network, and it does not participate in routing. 


\subsection{ZigBee Network Layer}

The ZigBee network layer defines how the network formation is performed (i.e., either mesh topology or tree topology) and how the network address is assigned to each participating ZigBee node. Note that the assigned network address is the only address that is used for routing and data transmission in ZigBee networks. Three device types are defined in ZigBee: ZigBee coordinator, ZigBee routers, and ZigBee end devices. A RFD can only be a ZigBee end device; whereas a FFD can be either a ZigBee coordinator or ZigBee router. The ZigBee coordinator is responsible for starting a new network. ZigBee coordinator and routers are "routing capable", while the ZigBee end devices can't participate in routing and have to rely on their corresponding ZigBee parent routers for that functionality.

Every node in a ZigBee network has two addresses, namely a 16-bit short network address and a 64-bit IEEE extended address. The 16-bit network address is assigned to each node dynamically by its parent coordinator/router upon joining the network. This address is the only address that is used for routing and data transmission. It is analogous to the IP addresses that we use on the internet; whereas the extended address is similar to the MAC address, which is a unique identification of each device and is mostly fixed at the time the device is manufactured.

\section{Mobility Support in ZigBee Mesh Topology}

As pointed out in section 2] only coordinators/routers (FFDs) can actively participate in mesh routing, end devices (RFDs) must rely on their parent nodes to perform mesh routing on their behalves. Under the innate properties of IEEE 802.15.4 and ZigBee networks (i.e., the addressing structure and service assumptions), the performance bound of ZigBee mesh routing is expected different to the ones from previous AODV studies. We will see the effect of this recovery mechanism in more detail in section 5 .

\subsection{Mobile End Device}

As mentioned earlier, ZigBee end devices are just simple devices without routing capabilities. Therefore, problems arise whenever these end devices moves out of the range of its parent router, and acquires a new network address from a new parent router, while the source node is still sending data to the mobile end device. Since this end device can no longer be found with its "old" address, data reception in this scenario will be halt completely, and can't be recovered from any available ZigBee mesh routing mechanisms. When the route cannot be found, a route error message will eventually be delivered to the source node, and trigger the Device Discovery primitive in the application layer. Once the source node discovers the new network address of the destination, the data transmission would resume (after another route discovery procedure). For this simple case, the data flow would only suffer the duration required for the source to receive the route error and complete its device discovery process.

For the case where the mobile end node acquires a new network address while it is sending data, data transmission will be temporally disrupted for the duration it takes for 
the mobile end node to find a new parent router to associate itself with. If the data flow is two way, a route discovery and Device Discovery process would be triggered at the receiver, and the disruption would be compounded, yet recoverable assuming that the mobile end device doesn't move out of range again.

\subsection{Mobile Router}

ZigBee routers actively participate in mesh routing, and provide functionalities that maintain/repair routes whenever an existing route failed. With the built-in route recovery mechanism (via route discovery and route error), ZigBee routers remains robust to effects from most mobility cases regardless whether the node is sending or receiving data. Once the router is assigned an initial network address, this is no explicit need to change this address.

\section{Mobility Support in ZigBee Tree Topology}

For ZigBee tree topology, the network address is assigned based on a hierarchical tree structure. As the root of the cluster tree, the ZigBee coordinator is responsible in defining the number children node a parent may have nwkMaxChildren $\left(C_{m}\right)$, the maximum number of routers a parent may have as children nwkMaxRouter $\left(R_{m}\right)$, and the maximum depth of the network nwkMaxDepth $\left(L_{m}\right)$. For a network of depth $d$, the $n^{\text {th }}$ network address is allocated according to Eq. 2and 1

$$
\begin{gathered}
C_{\text {skip }}(d)=\left\{\begin{array}{c}
1+C_{m}\left(L_{m}-d-1\right), R_{m}=1 \\
\frac{1+C_{m}-R_{m}-C_{m}\left(R_{m}\right)^{L_{m}-d-1}}{1-R_{m}}, \text { else }
\end{array}\right. \\
A_{n}=A_{\text {parent }}+C_{\text {skip }}(d) * R_{m}+n
\end{gathered}
$$

After network address is assigned, any node can then route packets to its parent and direct children with the tree routing algorithm. Trivially, every other device in the network is a descendant of the ZigBee coordinator and no device in the network is the descendant of any ZigBee end device. Each node would check the destination address against its own to determine if the destination is a descendent on the tree or if it should be forwarded to its parent node. When a node changes its parent router due to mobility, a new 16-bit network address will be automatically assigned to preserve the tree addressing structure. In many cases, simple mobility of a router can cause cascading address changes across entire tree branches. As we will discuss further in the evaluation, the Device Discovery service in application layer would be quite limited in accommodating some of the network changes, resulting in various levels of performance penalty.

\subsection{Mobile End Device}

By design, if the end device is mobile while transmitting data, it should resume the transmission as soon as it acquires its new network address. If the data flow is two way, a route discovery and Device Discovery process will be triggered at the receiver. If the 
end device is receiving data, the data flow would eventually recover if the application is successful in using the Device Discovery mechanism to rediscover the node's new network address. However, the Device Discovery mechanism would only work as intended under very limited mobility scenario (i.e., only one or two nodes moving within the network). As we will see in the subsequent section, when there are persistent or multiple occurrences of mobility, the longer routes and slower throughput (from multihopping) of tree routing tends to hinder the responsiveness of the described recovery scheme, causing a big degradation to performance.

\subsection{Mobile Router}

For ZigBee tree topologies, new network addresses are assigned in accordance to Eq. 2 to ensure the correct hierarchical tree structure. The stability of the addressing structure is important for the proper delivery of packets. Therefore, when ZigBee router acquires a new parent router and a new network address, it could potentially start a cascading network address change to all of its descendant nodes on impacted branches, which generally creates varying levels of inconsistency to the tree addressing scheme, thereby reducing the routing protocol's ability to function properly.

For the case that the mobile router moves out of the range of its original parent router and acquires a new network address, data reception will be halted completely, and cannot be recovered from any available ZigBee tree routing mechanisms. Sometimes, these simple movements would also jolt drastic structural change to the descendent nodes, influencing other existing flows. Even with the Device Discovery primitive in the application layer, the tree topology encounters great difficulties in recovering the path, since longer routes and slower throughput from tree routing tends to hinder the responsiveness of the described recovery scheme as mentioned earlier.

If the mobile router was sending data while it changes parent router, it would require its old descendants to change their network address. Depending on the number of impacted nodes, similar failure would be experienced as in the cases mentioned above. Except, the mobile router would probably be able to continue the data transmission once it acquires a new network address (in simple mobility scenarios). In any event, when there are persistent or multiple occurrences of mobility, the problem with tree routing would increase in complexity, and the tree routing will yield poor routing performances.

\section{Evaluation}

In this section, we present simulations results that illustrate the properties of ZigBee mesh and ZigBee tree routing schemes. We use the NS-2 simulator with Samsung's IEEE 802.15.4 extension [6], and contribute the implementation of the ZigBee tree routing and ZigBee mesh routing schemes according to the ZigBee standard version 1.0. The simulation is set to mimic the settings of a household/factory deployment. Nodes are initially aligned in an equally spaced grid before a selected percentage of nodes become mobile. Nodes move within the topology according to the random waypoint model [5], and all results are averaged across 10 independent trials of the same configuration. For all of our simulations, the network used in our simulation consists of 
Table 1. General Simulation Parameters

\begin{tabular}{|c|c||c|c|}
\hline Network Size & $45 \mathrm{~m} \times 45 \mathrm{~m}$ & Traffic Type, Packet Size & CBR, 127bytes \\
\hline Number of Nodes & 36 nodes & Mobility Model & Random Waypoint \\
\hline Transmission Range & 15 meters & $n w k M a x$ Router $\left(R_{m}\right)$ & 10 \\
\hline Network Setup Time & 30 seconds & Number of Concurrent Data Flows & 2 \\
\hline Simulation Duration & 300 seconds & $n w k M a x$ Depth $\left(L_{m}\right)$ & 5 \\
\hline Transmission Rate & 10 packets/sec & $n w k M a x$ Children $\left(C_{m}\right)$ & 10 \\
\hline
\end{tabular}

$70 \%$ routers and $30 \%$ end devices, which are all randomly chosen. We use packet delivery ratio and relative routing overhead as our performance evaluation matrices. Packet delivery ratio is averaged over the number of flows in the network to reflect the mean per-flow delivery ratio. On the other hand, routing overhead is denoted by a normalized value of the total overhead of the network with respect to the traffic in the network. The parameters employed in the simulation are summarized in Table 1

\subsection{Scenarios with Varying Percentage of Mobile Nodes}

This subsection studies the performance of the two ZigBee routing schemes when there are varying amount of mobile nodes in the network. Mobile nodes move at a speed of

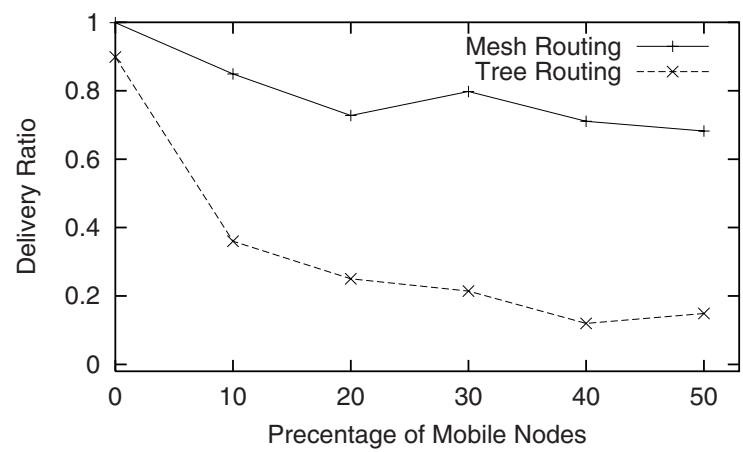

(a) packet delivery ratio

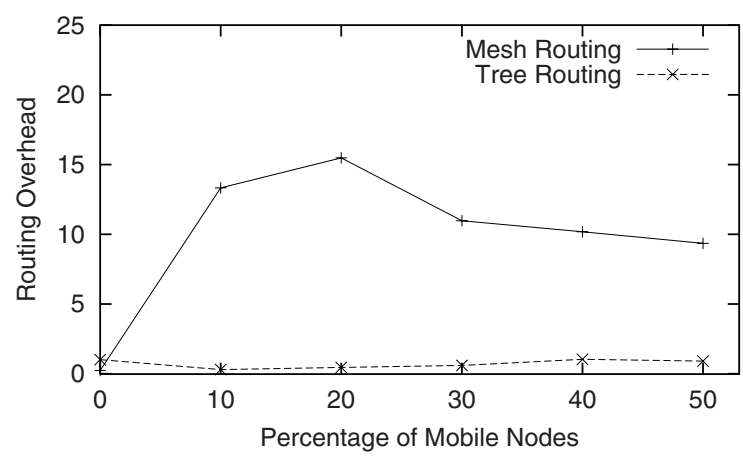

(b) relative routing overhead compared to the actual data throughput

Fig. 1. ZigBee router as mobile sender, data to stationary destination 


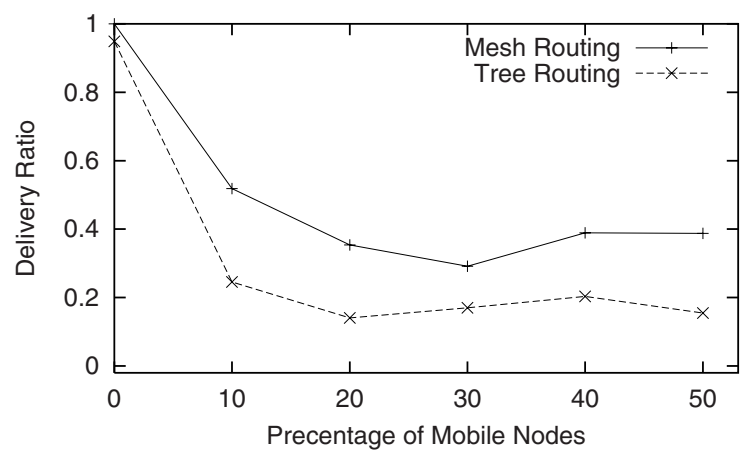

(a) packet delivery ratio

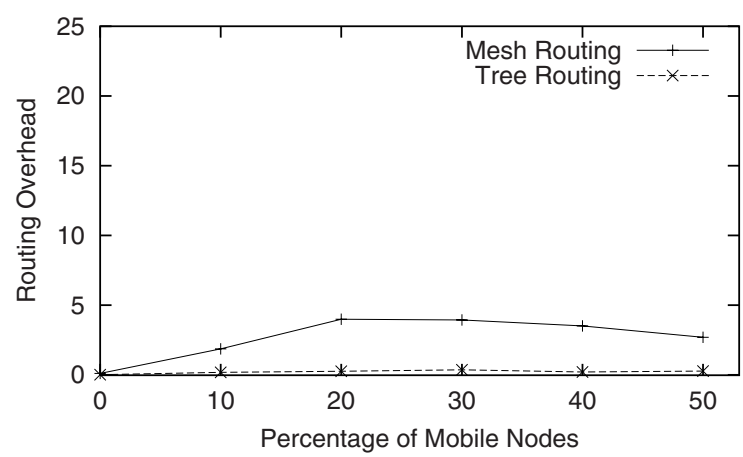

(b) relative routing overhead compared to the actual data throughput

Fig. 2. ZigBee end device as mobile sender, data to stationary destination

$1 \mathrm{~m} / \mathrm{s}$ randomly. Two general mobility cases were simulated. In the first scenario, the sender remains stationary while the receiver is mobile. In the second scenario, we keep the receiver stationary while setting senders to be mobile. We repeat the same simulations with two node types, i.e., ZigBee routers and end devices. Source and destination are randomly chosen, but all networking settings remain the same for all simulations. We vary the percentage of mobile nodes from 0 to $50 \%$ to observe the response from the two routing protocols to increasing percentages of mobile nodes in the network.

From the results depicted in Fig. 1 - a and 2 a, it is clear that the device type plays a critical role in determining the delivery ratio for mobile senders. ZigBee routers can typically transmit out more data, while ZigBee devices can only send out half of the amount compare to the routers. Furthermore, the ZigBee end devices are more heavily influenced by the percentage of mobile nodes in the network compare to the ZigBee router. This is due to the fact the ZigBee end devices need to associate with a new parent when it moves, the extra association time actually degrades the packet delivery ratio. On the other hand, from Fig. 1 - b and 2 b, we see that ZigBee routers actually incurs more routing overhead compare to the end devices. The additional routing overhead is from route repair messages that routers send/receive to repair the route. 


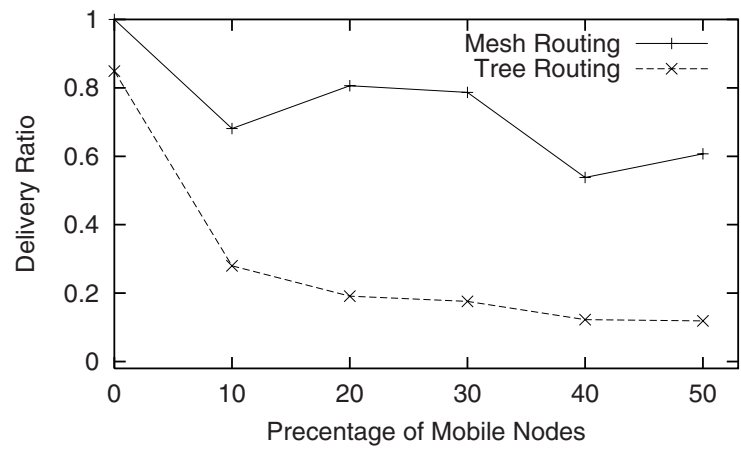

(a) packet delivery ratio

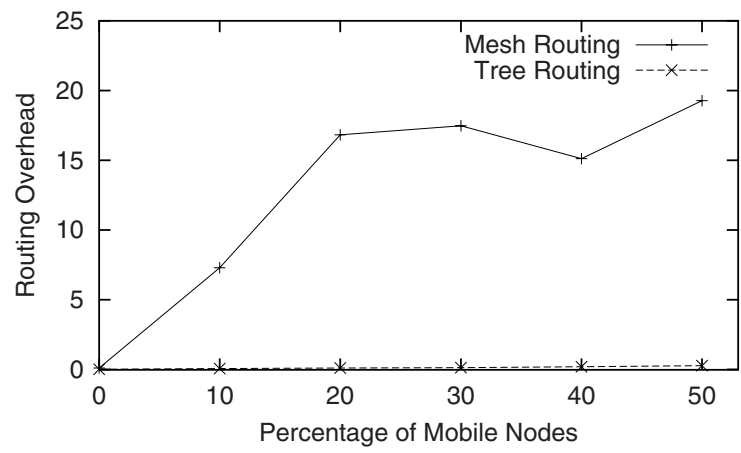

(b) relative routing overhead compared to the actual data throughput

Fig. 3. ZigBee router as mobile receiver, data from stationary source

Additionally, in Fig. 1 1 a and 2 a, we see that mesh routing exhibits better packet delivery ratio compared to tree routing. The performance gap is especially evident when the mobile sender is a ZigBee Router. It is clear that the rigid routing scheme demanded by tree routing is less robust to increasing amounts of mobility in the network, as it lacks an effective route recovery method when a route fails. Thus, delivery ratio suffers. When the network is experiencing multiple instances of mobility, it is apparent that the application recovery mechanism has a minimum effect in repairing broken routes. As a results, tree routing performs quite poor when the network comprises of $20 \%$ or more mobile nodes. Yet, as seen in Fig. 1 $\mathrm{b}$ and $2 \mathrm{~F}$, we see that tree routing incurs much smaller amount of routing overhead compare to mesh routing.

As the destination of data streams, all ZigBee receivers encounters some performance degradation (in terms of data delivery ratio) when it is mobile as illustrated in Fig. 3-a and 4-a. Device type actually differentiates the services received in the two routing schemes. Mobile receiver would clearly benefit if it is a ZigBee router in mesh routing. Nonetheless, device type would remain indifferent when tree routing is deployed, since movement in our tree topology would cause approximately the same amount of change regardless whether the node is a router or a end device. 


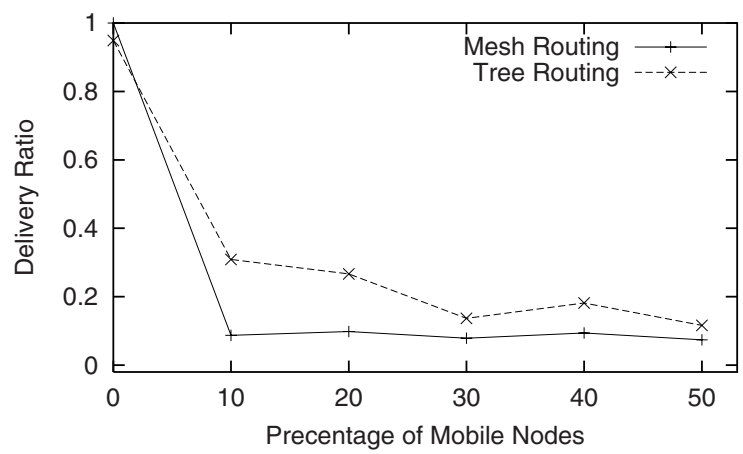

(a) packet delivery ratio

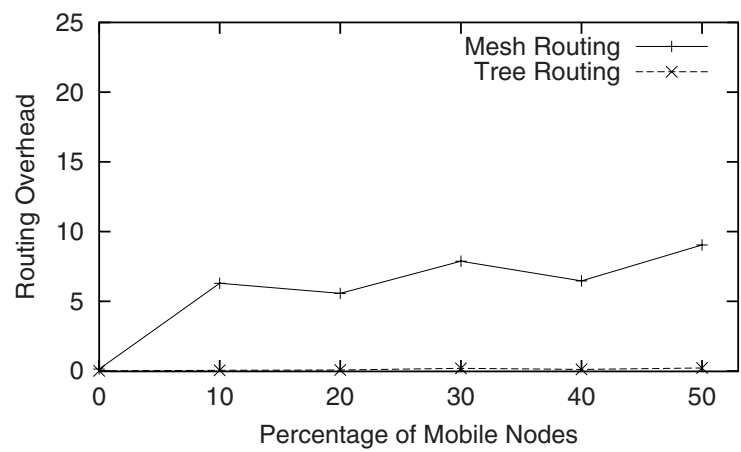

(b) relative routing overhead compared to the actual data throughput

Fig. 4. ZigBee end device as mobile receiver, data from stationary source

In Fig. 3 a, mesh routing obviously performs much better than tree routing since the route repair mechanism in mesh routing can repair some of the mobility induced damages. Results also confirm the intuition that mesh routing consumes more overhead when there are more mobile nodes in the network. It is also clear that tree routing consistently consumes less overhead than mesh routing, regardless of the number of mobile nodes in the network.

The only scenario that tree routing outperforms mesh routing is when ZigBee end devices are receivers. In this scenario, ZigBee end devices suffer degraded performance in mesh routing because it constantly acquires new network addresses, and the Device Discovery service cannot recover the new network address in a timely manner. However, since the ZigBee end device would pick the lowest ID node as its initial parent, tree routing tends to pick a parent node that is further away. Thus, when there are more mobile nodes in the network, there is a good chance to get closer to its original parent node. This explains the superior performance of tree over mesh routing in Fig. 4 . a.

The results in this subsection suggests the suitability of mesh routing for ZigBee network anticipating many instances of mobile nodes. Additionally, it shows that ZigBee routers tend have better delivery ratio in most scenarios. Plus, it shows that tree routing 
is the more effective scheme for static ZigBee networks with low data rate applications, due to its low overhead consumption.

\subsection{Scenarios with Mobile Nodes of Varying Speed}

Following the same methodology in the previous subsection, this subsection studies the routing performance of the two ZigBee routing schemes when the mobile nodes in the network are moving at varying speeds. The ZigBee network in question consist of $70 \%$ routers and $30 \%$ end devices, and $20 \%$ of the nodes in the network are selected randomly as mobile nodes. Specifically, we evaluate for packet delivery ratio when the nodes are moving from $1 \mathrm{~m} / \mathrm{s}$ to $5 \mathrm{~m} / \mathrm{s}$ in $1 \mathrm{~m} / \mathrm{s}$ increments.

Fig. 5-a and 6-a clearly show that the device type plays a critical role in determining the delivery ratio in mesh routing. ZigBee routers can typically transmit out more data, while ZigBee devices can only send out half of the amount compare to the routers. On the other hand, from Fig. 5-b and 6-b, we see that ZigBee routers actually incurs more routing overhead compare to the end devices. The additional

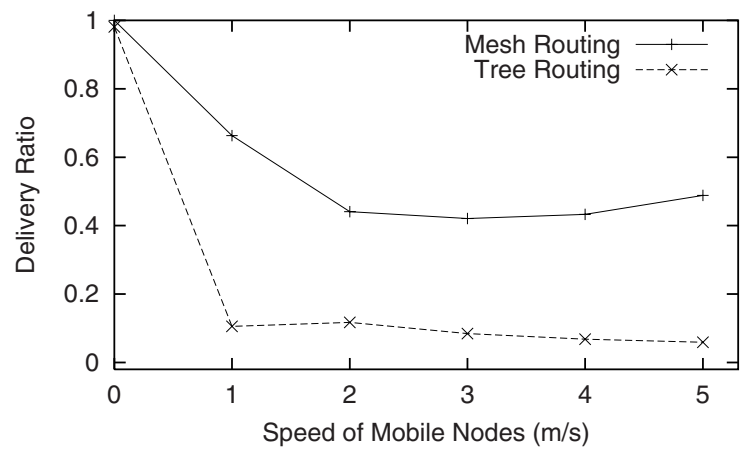

(a) packet delivery ratio

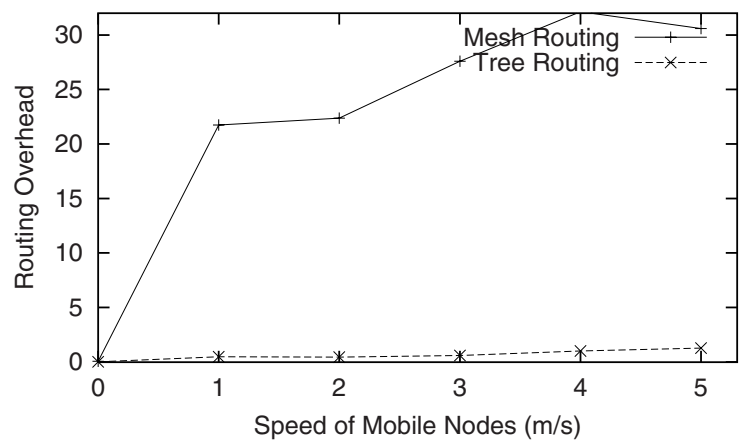

(b) relative routing overhead compared to the actual data throughput

Fig. 5. ZigBee router as mobile sender, data to stationary destination 


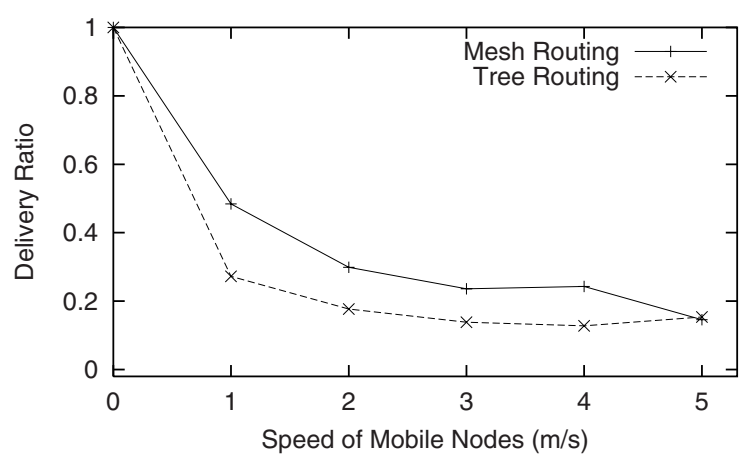

(a) packet delivery ratio

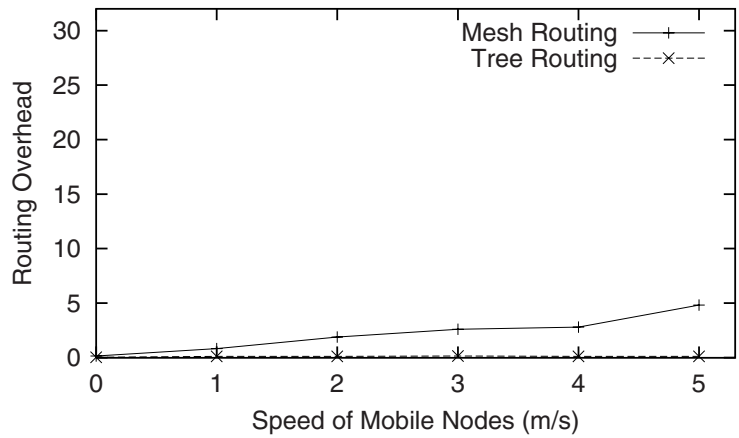

(b) relative routing overhead compared to the actual data throughput

Fig. 6. ZigBee end device as mobile sender, data to stationary destination

routing overhead is from the various route repair messages that routers send/receive to repair the route. We also see that as node speed increases, the delivery ratio decreases.

Additionally, in Fig. 5. a and 6,a, we see that mesh routing exhibits better packet delivery ratio than tree routing. Like in the previous subsection, the performance gap is especially evident when the mobile sender is a ZigBee Router. This is again due the ineffectiveness of tree routing's rigid routing scheme, and ZigBee router's ability to route for itself.The route repair mechanism in mesh routing makes them far more robust to mobility then their tree routing counterparts. When network speed increases, the application Device Discovery mechanism provided minimal help in reestablishing the route. As a results, we witness the same amount of performance degradation for the two tree routing scenarios. Yet, as seen in Fig. 5 -b and 6 -b, we see that tree routing again incurs much smaller amount of routing overhead compare to mesh routing.

In most scenarios, ZigBee receivers tend to encounters more severe performance degradation when it is traveling at higher speeds, as shown in Fig. 77-a and 8, a. As in the last subsection, device type actually differentiates the services received in the two 


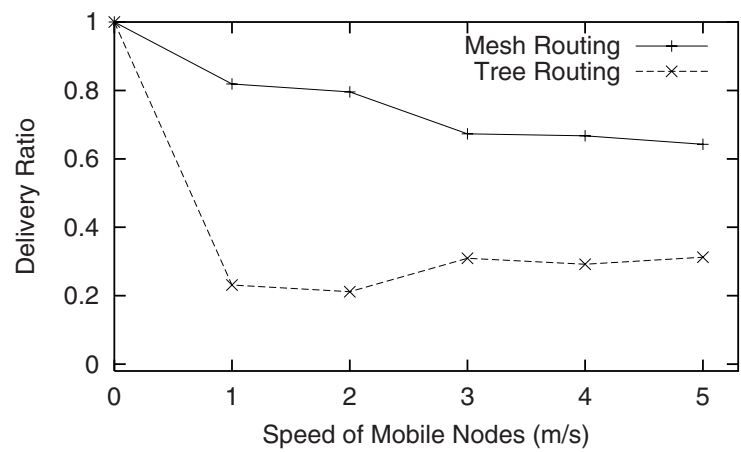

(a) packet delivery ratio

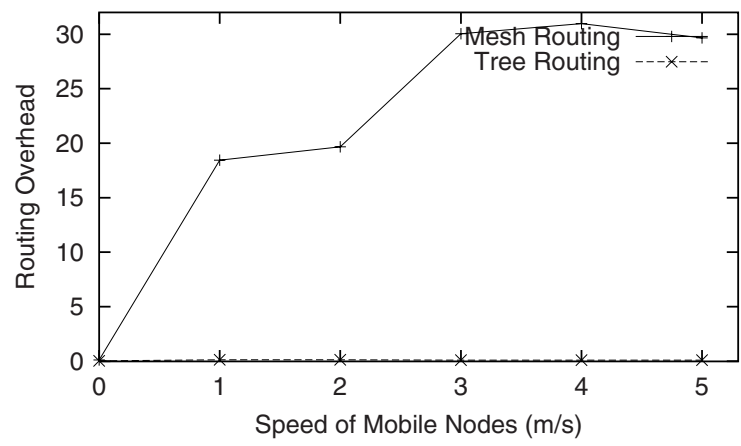

(b) relative routing overhead compared to the actual data throughput

Fig. 7. ZigBee router as mobile receiver, data from stationary source

routing schemes. Mobile receiver would clearly benefit if it is a ZigBee router in mesh routing. Nonetheless, device type would remain relatively indifferent when tree routing is deployed, for the same reason pointed out earlier.

As depicted in Fig. 7 a, mesh routing with ZigBee routers exhibited more resiliency against high node speeds, even though it consumes more overhead than tree routing as illustrated in Fig. 7 $\mathrm{b}$ and 8-b. Like in the previous subsection, the only scenario that tree routing outperforms mesh routing is when ZigBee end devices are receivers. The reason is the same as in the previous subsection, and it also illustrates the inefficiency of the application Device Discovery mechanism in recovering the route.

The overall results in this subsection suggest the suitability of mesh routing for ZigBee networks anticipating high speed mobile nodes. It also shows that tree routing is more effective for static ZigBee network with low rate applications, due to its low overhead consumption (and saves more energy). However, mesh routing is clearly more robust to nodal mobility, which closes echoes the findings in previous sections. 


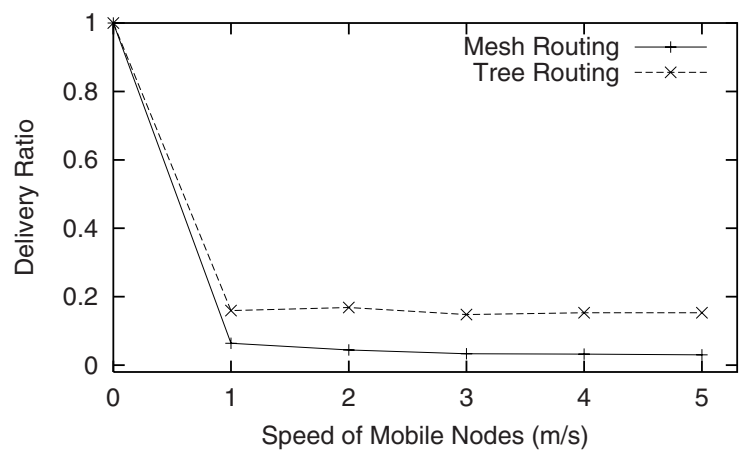

(a) packet delivery ratio

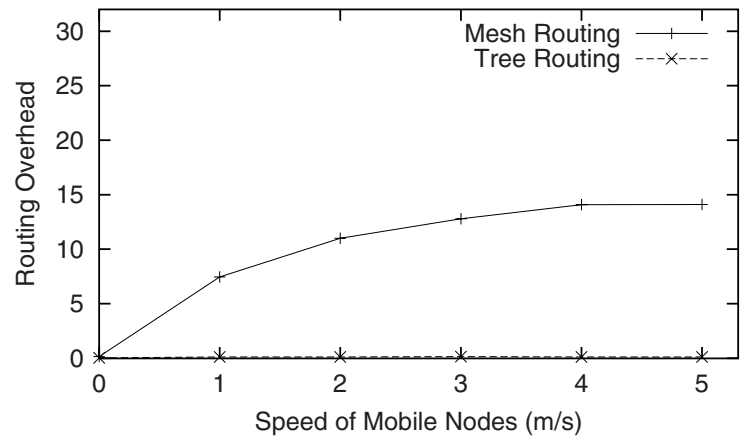

(b) relative routing overhead compared to the actual data throughput

Fig. 8. ZigBee end device as mobile receiver, data from stationary source

\section{Conclusion}

In this study, we discussed ZigBee routing and its support for device mobility, and we analyzed the adequacy of current provisions in dealing with different mobility cases. Our evaluation results indicate that when network is static, both mesh and tree routing schemes work as intended; however, ZigBee end devices experiences detrimental packet losses in almost all mobility scenarios. This situation worsens under multiple instances of mobility, and when mobile nodes travel at higher speeds. Yet, ZigBee router typically suffers less packet losses under mobile scenarios. This behavior is closely related the fact that ZigBee router are routing capable, while the ZigBee end devices are not. We also realized that the current recovery mechanism is inadequate in accommodating multiple instance or rapid mobility. Additional design work is underway to resolve the various problems pointed out in this paper.

\section{References}

1. Ieee 802.15.4. http: / / www . ieee802 .org/15/pub/TG4 .html

2. Zigbee specification v1.0 (June 2005) 
3. Jafari, R., Encarnacao, A., Zahoory, A., Dabiri, F., Noshadi, H., Sarrafzadeh, M.: Wireless sensor networks for health monitoring. In: ACM/IEEE ICMUS (2005)

4. Korhonen, I., Parkka, J., Gils, M.V.: Health monitoring in the home of the future. In: IEEE EMBM (2003)

5. PalChaudhuri, S., Boudec, J.-Y.L., Vojnovic, M.: Perfect simulations for random trip mobility models. In: 38th Annual Simulation Symposium (2005)

6. Zheng, J., Lee, M.J.: A Comprehensive Preformance Study of IEEE 802.15.4. In: Sensor Network Operations, ch. 4, pp. 218-237. IEEE Press, Los Alamitos (2006) 